\title{
FEF-Controlled Alpha Delay Activity Precedes Stimulus-Induced Gamma-Band Activity in Visual Cortex
}

\author{
(DTzvetan Popov, ${ }^{1}$ Sabine Kastner, ${ }^{2,3}$ and ${ }^{\mathbb{C}}$ Ole Jensen ${ }^{4,5}$ \\ ${ }^{1}$ Department of Psychology, University of Konstanz, Konstanz 78462, Germany, 2Princeton Neuroscience Institute and ${ }^{3}$ Department of Psychology, Princeton \\ University, Princeton, New Jersey 08544, ${ }^{4}$ Donders Institute for Brain, Cognition and Behaviour, Radboud University, Kapitelweg 29, Nijmegen 6525 EN, The \\ Netherlands, and 5 School of Psychology, Centre for Human Brain Health, University of Birmingham, Hills Building, Birmingham B15 2TT, United Kingdom
}

Recent findings in the visual system of nonhuman primates have demonstrated an important role of gamma-band activity $(40-100 \mathrm{~Hz})$ in the feedforward flow of sensory information, whereas feedback control appears to be established dynamically by oscillations in the alpha $(8-13 \mathrm{~Hz})$ and beta $(13-18 \mathrm{~Hz})$ bands (van Kerkoerle et al., 2014; Bastos et al., 2015). It is not clear, however, how alpha oscillations are controlled and how they interact with the flow of visual information mediated by gamma-band activity. Using noninvasive human MEG recordings in subjects performing a visuospatial attention task, we show that fluctuations in alpha power during a delay period in a spatial attention task preceded subsequent stimulus-driven gamma-band activity. Importantly, these interactions correlated with behavioral performance. Using Granger analysis, we further show that the right frontal-eye field (rFEF) exerted feedback control of the visual alpha oscillations. Our findings suggest that alpha oscillations controlled by the FEF route cortical information flow by modulating gamma-band activity.

Key words: alpha gamma oscillations; executive control; Flanker task; functional connectivity; magnetoencephalography; prefrontal control

\section{Significance Statement}

Visual perception relies on a feedforward flow of information from sensory regions, which is modulated by a feedback drive. We have identified the neuronal dynamics supporting integration of the feedforward and feedback information. Alpha oscillations in early visual regions reflect feedback control when spatial attention is allocated and this control is exercised by the right frontal eye field. Importantly, the alpha-band activity predicted both performance and activity in the gamma band. In particular, gamma activity was modulated by the phase of the alpha oscillations. These findings provide novel insight into how the brain operates as a network and suggest that the integration of feedforward and feedback information is implemented by cross-frequency interactions between slow and fast neuronal oscillations.

\section{Introduction}

Converging evidence from studies in humans and nonhuman primates suggests that the feedforward drive of sensory information is accompanied by neuronal synchronization in the gamma band (van

Received Sept. 27, 2016; revised Feb. 13, 2017; accepted Feb. 20, 2017.

Author contributions:T.P., S.K., and 0.J. designed research; T.P. performed research; T.P. and 0.J. analyzed data; T.P., S.K., and 0.J. wrote the paper.

T.P. was supported by the Netherlands Organization for Scientific Research (NWO VICI Grant 453-09-002 to 0.J.) S.K. was supported by the National Institutes of Health (Grants R01-MH64043 and R01-EY017699), the National Science Foundation (Grant BCS-132827), and the James S. McDonnell Foundation Understanding Human Cognition Collaborative Award 220020448. We thank Yuri Saalmann and Liang Wang for providing a version of the code for the Flanker paradigm.

The authors declare no competing financial interests.

Correspondence should be addressed to Ole Jensen, School of Psychology, Centre for Human Brain Health, University of Birmingham, Hills Building, Birmingham B15 2TT, UK. E-mail: 0.jensen@bham.ac.uk.

DOI:10.1523/JNEUROSCI.3015-16.2017

Copyright $\odot 2017$ the authors $\quad 0270-6474 / 17 / 374117-11 \$ 15.00 / 0$
Kerkoerle et al., 2014; Michalareas et al., 2016). This neuronal synchronization is thought to promote synaptic summation, thereby fostering information transfer (Tiesinga et al., 2004). The feedback control of visual processing is often studied in the framework of spatial attention (Liu et al., 2016) and feedback mechanisms have been associated with modulations of alpha and beta oscillations (Worden et al., 2000; Kelly et al., 2006; Palva and Palva, 2007; Rihs et al., 2007; Bahramisharif et al., 2010; Palva and Palva, 2011; Bauer et al., 2014; Lozano-Soldevilla et al., 2014; Marshall et al., 2015b). Although different frequency ranges might provide different channels for communicating feedforward and feedback information, the neuronal dynamics supporting the integration of information remain unknown.

Consistent with the notion of feedback, alpha oscillations typically decrease in power in anticipation of incoming stimuli, whereas the onset of, for example, a visual stimulus is associated with an increase in induced gamma power (Foxe et al., 1998; Thut et al., 
2006; Bauer et al., 2014; Lozano-Soldevilla et al., 2014; Marshall et al., 2015b). In the past, it has been suggested that the decrease of alpha power ("desynchronization") reflects engagement (Pfurtscheller, 1977; Pfurtscheller and Aranibar, 1979). Importantly, whereas alpha power is decreased, the phase of the alpha oscillations appears still to be functional (van Kerkoerle et al., 2014; Dougherty et al., 2015). It has been suggested that the reduction of the inhibitory alpha oscillations allows for a longer duty-cycle, thus allowing for more information to be processed (Jensen et al., 2014). Results from a recent monkey physiology study suggest that interregional alpha band synchronization between cortical areas and the pulvinar is associated with the allocation of visuospatial attention (Saalmann et al., 2012). Consistent with this notion, Dougherty et al. (2015) made a strong case for spike timing "clocked" by the phase of ongoing alpha activity in visual cortex. In addition, a recent ECoG study in humans suggested that phase-amplitude coupling served to organize a "phase code" transmitting categorical information about object representations (Watrous et al., 2015). These findings suggest that alpha oscillations are intimately involved in routing mechanisms controlling the feedforward drive implemented by gamma-band synchronization. Here, we consider several open questions that result from this framework: (1) does alpha-band activity reflect a feedback drive?, (2) which frontal areas regulate the feedback that exerts the top-down control reflected in the alpha band?, and (3) are the phase and amplitude of alpha oscillations in sensory regions predictive of subsequent stimulus-induced gamma-band activity?

\section{Materials and Methods}

Participants. Thirty participants ( 15 females, mean age $27.7 \pm 8.8$ years) who reported no history of neurological and/or psychiatric disorders were recruited. All participants gave written informed consent before the experiment in accordance with the Declaration of Helsinki. The study was approved by the local ethical committee (commission for human related research CMO-2014/288 region Arnhem/Nijmegen NL).

Stimulus material and procedure. A modified version of the "EriksenFlanker" design was used (Saalmann et al., 2012). In each trial, participants were instructed to fixate on a central white square $\left(0.82^{\circ}\right.$ visual angle) presented on a gray background at a distance of $\sim 84 \mathrm{~cm}$. During baseline "fixation" periods of $2 \mathrm{~s}$ (see Fig. $1 A$ ), they were instructed to blink if they needed to. The participants then covertly attended (while maintaining fixation) to a spatial cue (100 ms duration; $1.36^{\circ}$ visual angle), which appeared randomly at 1 of 16 locations (see Fig. $1 A$ ). After a delay interval of $2.5 \pm 1 \mathrm{~s}$, the target appeared at the precued location in a circular array of 16 stimuli (radius $16.5 \mathrm{~cm}$, visual angle $22.22^{\circ}$ ). Participants indicated via button press whether a target shaped as a "barrel" (left index finger) or a "bowtie" (right index finger) was shown. Targets were flanked either by congruent (C) or incongruent (IC) distracters. Targets and distracters had a visual angle of $2.73^{\circ} \times 3.07^{\circ}$. Target type (barrel or bowtie, $50 \%$ chance), as well as distractor congruency (C or IC, $50 \%$ chance), were determined randomly on each trial.

The visual stimuli were back projected onto a semitranslucent screen by an EIKI LC-XL100L projector with a projection resolution of $1024 \times$ 768 pixels. Eye movements and blinks were recorded continuously using an Eyelink 1000 eye-tracking device (SR Research).

Data acquisition. Neuromagnetic activity was monitored by a wholehead 275-sensor axial-gradiometer system (Omega 2000; VSM MedTech). The data were sampled at $1.2 \mathrm{kHz}$ after a $400 \mathrm{~Hz}$ low-pass filter was applied. After the MEG session, anatomical MRI images were acquired using a 1.5 T Siemens Magnetom Avanto system.

Neural data analysis. The data analysis was performed with the Matlab FieldTrip toolbox (Oostenveld et al., 2011) complemented by customwritten software. Trials that were contaminated by saccades due to subjects breaking fixation were excluded from subsequent analysis. Epochs contaminated by strong muscle movement and/or sensor "jump" artifacts were rejected from further analysis based on visual inspection. On average, $519 \pm 92$ of 640 ( 40 per location) trials were used in the analysis
(226 \pm 41 during right and $226 \pm 41$ during left attention conditions). Trials in which attention was directed toward "up" and "down" were not analyzed ( $34 \pm 6$ for attention "up" and $33 \pm 6$ for attention "down"). An independent component analysis (ICA) (Jung et al., 2001) was applied after demeaning and removing the linear trend from the data. Components associated with eye blink and cardiac activity were removed using the ICA approach.

Spectral analysis. Spectral analysis was computed for each trial using a fast Fourier transformation (FFT) approach. For frequencies $<30 \mathrm{~Hz}$, we used a sliding window approach ( $500 \mathrm{~ms}$ long) multiplied by a Hanning taper. The time window advanced in $50 \mathrm{~ms}$ increments and the spectral resolution was $2 \mathrm{~Hz}$. For frequencies $>30 \mathrm{~Hz}$, spectral analysis was done using a multitaper approach with orthogonal Slepian tapers (Mitra and Pesaran, 1999). Eleven orthogonal Slepian tapers were used, resulting in a frequency smoothening of $\pm 10 \mathrm{~Hz}$.

Spectral power was calculated for the horizontal and vertical components of the estimated planar gradient for each sensor location and subsequently summed (Bastiaansen and Knösche, 2000). This step usually simplifies the interpretation of the signal topography because maxima typically are observed above a given source (Hämäläinen et al., 1993). Analyses of oscillatory power were performed at sensor level for two reasons: comparability of findings with previous literature and sampling of multiple distributed activity primarily within parieto-occipital cortex linearly superimposed on the level of the sensors. Connectivity analyses [i.e., cross-frequency coupling and Granger causality (GC), see next section] were performed at source level to minimize the influence of spurious connectivity estimates (Bastos and Schoffelen, 2015).

Spectral estimates of sensor level data are reported as a comparison (attention left vs attention right) according to the following operation:

relative power change $=\left(\right.$ power $_{\text {left attention }}-$ power $\left._{\text {right attention }}\right)$

$$
/\left(\text { power }_{\text {left attention }}+\text { power }_{\text {right attention }}\right)
$$

See also the "Statistical analysis" section.

Quantification of saccade direction. Saccades were analyzed using the algorithm described in Engbert and Kliegl (2003). Epochs around the delay interval ranging from 500 to $1500 \mathrm{~ms}$ after cue onset were selected and epochs dominated by eye-blink artifacts were excluded. Eye-tracking data from three participants were excluded due to poor data quality. For each participant, epochs contaminated by vertical eye movements (blinks) were first excluded from further analysis, resulting in $212 \pm 60$ trials for attention directed to the left and $213 \pm 61$ trials for attention directed to the right hemifield. On average, $37.9 \%$ of the trials with attention directed to the left and $37.2 \%$ with attention directed to the right hemifield were characterized by pronounced macrosaccades (based on visual inspection). Microsaccades and macrosaccades were quantified on the basis of the angular eye velocity. A combined threshold of horizontal and vertical components was used to identify saccadic eye movements equal to six times the median SD of the velocity within a given epoch. The minimum duration was set to four samples (13 ms). A Kolmogorov-Smirnov test was used to test condition differences in saccade direction.

Measures of lateralization for between and within-subject evaluation. For between-subject evaluation, hemispheric lateralization (see Fig. $4 A, B, D$ in Results section) was estimated as follows:

$$
\begin{aligned}
\text { hemispheric laterlization }= & \left(\text { power left attention }_{\text {left sensor }}^{\text {lewer }}-\text { powt attention }_{\text {right sensor }}^{\text {ref }}\right) \\
& -\left(\text { power }_{\text {right attention }}^{\text {left sensor }}-\text { power }_{\text {right attention }}^{\text {right sensor }}\right)
\end{aligned}
$$

For within-subjects evaluation, for each trial and attention condition (left and right), the lateralization index was computed using the following equation: $\mathrm{LI}_{\alpha}=10^{\star} \log 10$ ( $_{\text {power }}$ ipsi $/$ power $_{\text {contra }}$ ) for occipital sensors selected by a permutation approach (see "Statistical analysis" section), where $\mathrm{LI}_{\alpha}$ is the lateralization index in the alpha band. These trials were divided into two pools according to low and high alpha-lateralization for the left and right attention conditions within participants. The $\mathrm{LI}_{\alpha}$ was 
therefore different from 0 (see Fig. 4C, left bars). For these two pools of trials, we computed LI in the gamma band during the target interval as follows: $\mathrm{LI}_{\gamma}=10^{\star} \log 10$ ( power $_{\text {ipsi }} /$ power $_{\text {contra }}$ ).

Source analysis. Source estimates were computed applying a frequencydomain adaptive spatial filtering algorithm (dynamic imaging of coherent sources; Gross et al., 2001). This algorithm uses the cross-spectral density (CSD) matrix from the MEG data and the lead field derived from the forward model to construct a spatial filter for a specific location ("voxel"). This CSD was calculated using a multitaper FFT approach for data in the $0.3-1.5$ $s$ target interval. Spectral smoothening of $\pm 2 \mathrm{~Hz}$ around a center frequency of $10 \mathrm{~Hz}$ was applied using 3 Slepian tapers, effectively including the power in the $8-12 \mathrm{~Hz}$ range, thus providing a good estimate of the classical $8-13 \mathrm{~Hz}$ band that typically defines the alpha band. These spatial filters were estimated on the basis of all trials. After identification of the fiducials, the nasion, and the left and right preauricular points, coregistration with Montreal Neurological Institute (MNI) coordinates was applied. A realistic, single-shell brain model (Nolte, 2003) was constructed based on the anatomical MRI. Forward solution for each participant was estimated using a common dipole grid (10 $\mathrm{mm}^{3}$ grid) in MNI space warped onto each participant's anatomy.

Cross-frequency analysis. The data were segmented to include the delay interval of -1 to $0 \mathrm{~s}$ relative to the array onset. These epochs were then concatenated to form a data matrix of $m$ sensors by $n$ time points. Data during the encoding interval were not considered for the analysis of phase-to-amplitude coupling (PAC) due to the presence of strong evoked activity. Before concatenation, epochs were multiplied with a Hanning taper. The concatenated trials were band-pass filtered at 8-12 $\mathrm{Hz}$ and the alpha-band troughs identified. Subsequently, $1 \mathrm{~s}$ epochs phase aligned to the troughs were extracted from the unfiltered data. Finally, time-frequency representations (TFRs) of power were calculated for each of these trough-locked epochs using Moret wavelets (TallonBaudry and Bertrand, 1999) with seven cycles width and then averaged across epochs. This allowed for quantifying how gamma power was modulated by alpha phase. Condition differences in cross-frequency coupling were evaluated by comparing PAC during left versus right attention conditions using nonparametric randomization approach (see below).

GC analysis. Spectrally resolved GC analysis (Granger, 1969; Ding et al., 2006; Wen et al., 2013) was used to dissociate the directionality (i.e., feedforward vs feedback influences) of information flow between cortical areas. The time series were projected to source space by applying a timedomain spatial filtering algorithm (linearly constrained minimum variance) (Van Veen et al., 1997). This algorithm uses the covariance matrix of the MEG data to construct a spatial filter for a given location (voxel). These spatial filters were estimated on the basis of all trials and for the -1 to $0 \mathrm{~s}$ interval just before stimulus onset. Subsequently, these filters were applied to the data to estimate the time series for a given location. The source level time-series were first down-sampled to $200 \mathrm{~Hz}$ and resegmented to include a $\pm 1 \mathrm{~s}$ time window around the stimulus array onset. A bivariate nonparametric spectral matrix factorization approach (Wen et al., 2013) was applied to estimate the GC. This algorithm estimates the spectral density matrix on the basis of the Fourier coefficients. The Fourier coefficients were computed using multitapers as described above (3 orthogonal Slepian tapers were used) with frequency smoothening of \pm 3 $\mathrm{Hz}$ for frequencies of $0-100 \mathrm{~Hz}$ with a $1 \mathrm{~Hz}$ spectral resolution. On the basis of the spectral density matrix (i.e., applying spectral factorization), the noise covariance matrix and the transfer function were obtained (Wen et al., 2013). Two seed locations were chosen in bilateral primary visual areas, V1/BA17 $(28,-96,-6 \mathrm{~mm} ;-28 \mathrm{~mm},-96 \mathrm{~mm},-6 \mathrm{~mm})$. These seed regions were selected on the basis of the source analysis results using a cluster permutation approach (see Fig. 4A). The GC estimates were computed for all voxels and the seeds in V1 in both directions.

Statistical analysis. Statistical quantification of the neuronal data was performed by a cluster-based approach based on randomizations (Maris and Oostenveld, 2007). This approach identifies clusters of activity on the basis of whether the null hypothesis can be rejected while controlling for multiple comparisons. The test statistic for a given condition $\mathrm{A}$ and $\mathrm{B}$ was built on the basis of relative changes (see Fig. 2) defined as follows: test statistic $=$ $(A-B) /(A+B)$ (Lozano-Soldevilla et al., 2014; Spaak et al., 2014).

Effects of congruency and visual field location on behavioral responses were assessed by an ANOVA approach with the factors congruency (C,
IC) and hemifield (left, right). Significant main effects and interactions were decomposed by simple-effects ANOVA or $t$ test. Relationships between behavioral and neuronal data were examined using Spearman's rank-order correlations $(\rho)$.

\section{Results}

\section{Behavioral Flanker effects: increases in reaction time and decreases in accuracy}

MEG data were recorded from 30 participants performing an Eriksen-Flanker task (Saalmann et al., 2012; Martin et al., 2015); Fig. 1A). After a $2 \mathrm{~s}$ fixation period, a spatial cue was presented for $0.1 \mathrm{~s}$ indicating the location of an upcoming target, which was shown for $0.7 \mathrm{~s}$ after a variable delay period $(2.5 \pm 1 \mathrm{~s})$ in that location embedded in a circular array of 16 stimuli. Participants had to indicate whether the target was a barrel or a bowtie shape with a button press of the right or left index finger, respectively. Targets at the cued position were either flanked by C or IC stimuli.

As shown in previous studies on the Eriksen-Flanker task (Eriksen and Eriksen, 1974; Eriksen, 1995), targets flanked by IC distracters were associated with a decrease in accuracy (Fig. $1 B$, left); this effect was observed in both hemifields (left hemifield: $t_{(29)}=-9.3, p<0.001$; right hemifield, $t_{(29)}=-11.01, p<$ 0.001 ). Moreover, reaction times (RTs) increased after presentation of IC compared with $\mathrm{C}$ distracters (Fig. $1 B$, right; left hemifield: $t_{(29)}=6.4, p<0.001$; right hemifield, $t_{(29)}=8.7, p<0.001$ right) and there was no congruency $\times$ hemifield interaction $(F<$ $1)$. Therefore, we replicated the core effects of the Flanker paradigm by showing that IC flankers impaired performance by increasing RTs and decreasing accuracy.

\section{Alpha power during the delay period predicts stimulus- induced gamma power}

A time-resolved spectral analysis was applied to the MEG data. Trials were divided according to whether the targets were cued to the left or the right visual hemifield. Figure $2 A$ illustrates the TFRs aligned to target onset $(t=0 \mathrm{~s}$; TFRs averaged over sensors marked in Fig. $2 A$, right). When comparing trials with cues in the left versus right visual hemifield, the alpha power increased in anticipation of the target array over the left hemisphere, whereas it decreased over the right hemisphere. The topography (Fig. 2A, right) shows the $8-13 \mathrm{~Hz}$ alpha-band modulations (left vs right cued trials) before stimulus onset ( -1 to $0 \mathrm{~s})$. This period was chosen to avoid contamination by sensory cue $(\sim-1.5 \mathrm{~s})$ before stimulus onset during the delay interval (Fig. $1 A$ ). The marked sensors indicate clusters of significant alpha-band modulation; that is, power modulations between left and right cued trials $(p<0.05$; multiple comparisons over sensors was controlled using a cluster-based randomization approach; Maris and Oostenveld, 2007). Analysis of eye movements revealed no condition differences in saccade direction in relation to the cuing during the delay interval (Fig. 2C).

The same analysis was then applied to higher frequencies aligned to target onset (Fig. $2 B$ ). The target array induced gamma-band activity (60-80 Hz) (Müller et al., 2000; Fries et al., 2001; Siegel et al., 2008; Lozano-Soldevilla et al., 2014; Marshall et al., 2015b) that was modulated robustly by the direction of attention. The left cue prompted a sustained gamma power increase in right sensors and a relative decrease in left sensors. The corresponding topography of this relative effect is shown in Figure $2 B$ ( $p<0.005$; cluster-based permutation test).

We next investigated to what extent the phase of the ongoing alpha activity modulated the amplitude in the gamma band. We calculated a PAC measure for each condition within bilateral V1 (same virtual sensor locations as for the GC analysis; see last section 

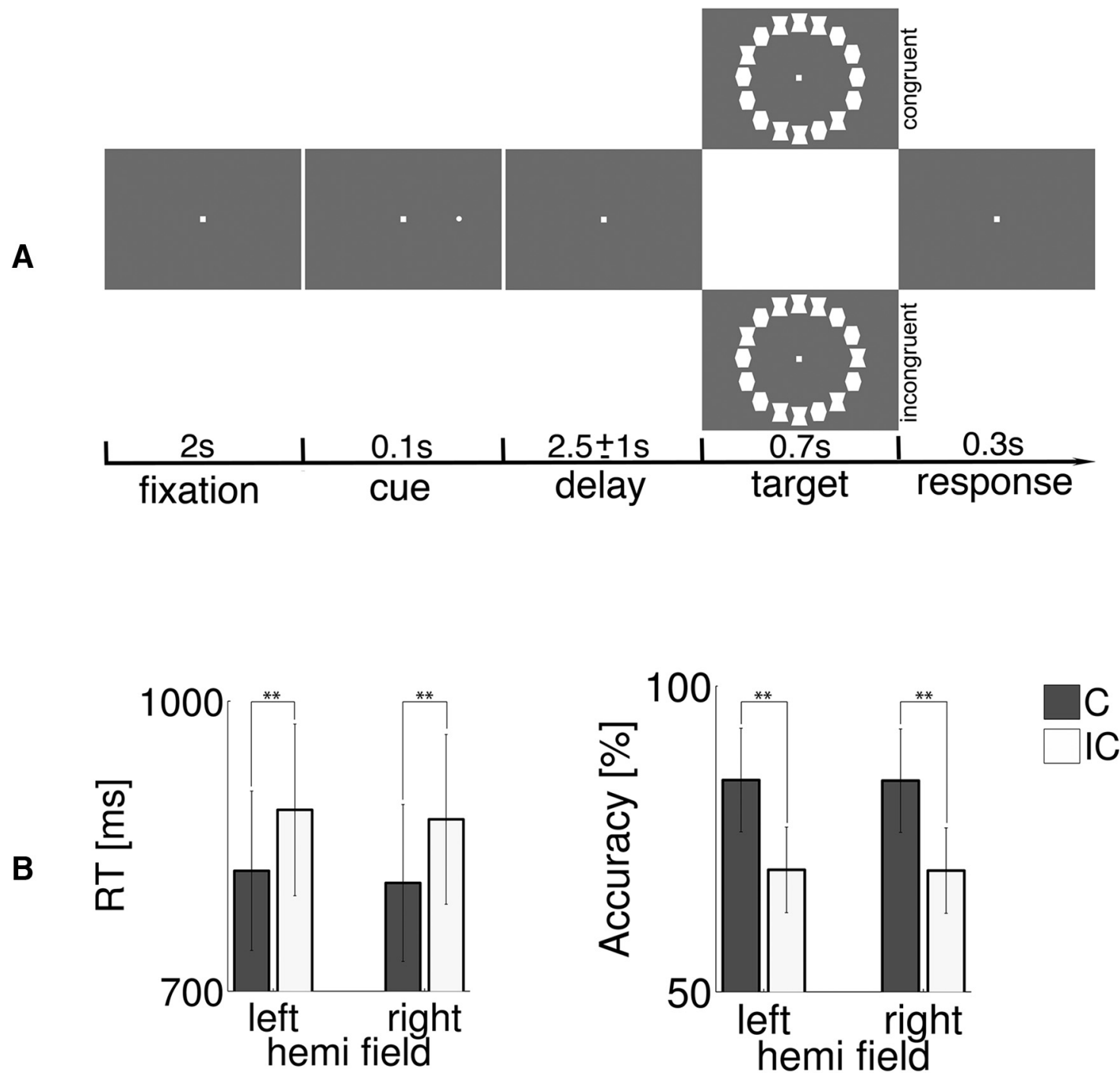

Figure 1. Experimental design and behavioral outcome. $\boldsymbol{A}$, After a fixation interval $(2 \mathrm{~s})$, a visual cue was presented $(0.1 \mathrm{~s})$ in one of 16 locations, followed by a delay (cue-target) interval of 0.1-3.5 s during which the cue location needed to be remembered. Upon presentation of a circular stimulus array, participants had to indicate whether the target at the cued location was a bowtieor a barrel-shaped stimulus. Target stimuli were flanked by either C or IC stimuli. B, RTs (left) were faster for targets flanked by C (gray bars) compared with IC (white bars) flanking stimuli; this effect was similar in both visual hemifields. Likewise, accuracy was better for C compared with IC flankers (accuracy; right). Error bars indicate SEM.

in Results). Subsequently, a nonparametric cluster based statistical evaluation was performed comparing attend-left versus attend-right PAC within each of the two visual areas. During the delay interval, condition-specific modulation of gamma activity $(60-80 \mathrm{~Hz})$ as a function of the alpha phase was found (Fig. $3 A$, right; $p<0.05$ nonparametric cluster-based permutation test controlling for multiple comparisons over time and frequency when comparing left vs right hemifield attention conditions). There was a significant relationship between attention-related (left vs right attention) PAC modulation and behavioral performance (Fig. $3 B$, middle and right). Gamma power was less modulated in the hemisphere ipsilateral to the direction of attention and did not relate significantly to performance (Fig. $3 A, B$, left). In short, during the delay interval, attention deployment prompted a contralateral decrease of alpha activity. Subsequent stimulus processing was associated with a contralateral increase in gamma activity. There was a robust cross-frequency relationship between alpha phase and gamma amplitude in the delay interval in both hemispheres. The magnitude of the coupling was somewhat higher in the right hemisphere. A stronger gamma amplitude reduction by the alpha phase was associated with shorter RTs.
We then investigated whether the modulations in alpha and gamma power predicted behavior as measured by the degree of distractibility by IC flankers $\left(\Delta \mathrm{RT}=\mathrm{RT}_{\mathrm{IC}}-\mathrm{RT}_{\mathrm{C}}\right)$. We correlated the hemispheric alpha lateralization with the difference in $\Delta \mathrm{RT}$ over subjects. Reaction times were less affected by IC flankers (Fig. $4 A$, left; $\rho=-0.68, p<0.001$ ) in subjects with stronger alpha lateralization, suggesting more effective filtering of distracter information. In addition, stronger stimulus-induced gamma-band lateralization was correlated with change in RT (Fig. $4 B$, right; $\rho=$ p.54, $p<0.01$ ). These behavioral results indicate that both prestimulus alpha activity and stimulusinduced gamma activity can be predictive of behavioral outcome and therefore are likely to be coupled. We probed the nature of this coupling next.

Fluctuations in alpha modulation predict gamma activity To test the relationship between the feedback-driven prestimulus alpha-band activity and the stimulus-induced gamma-band activity, the lateralization index (see "Measures of lateralization for between and within-subject evaluation" section in the Materials 

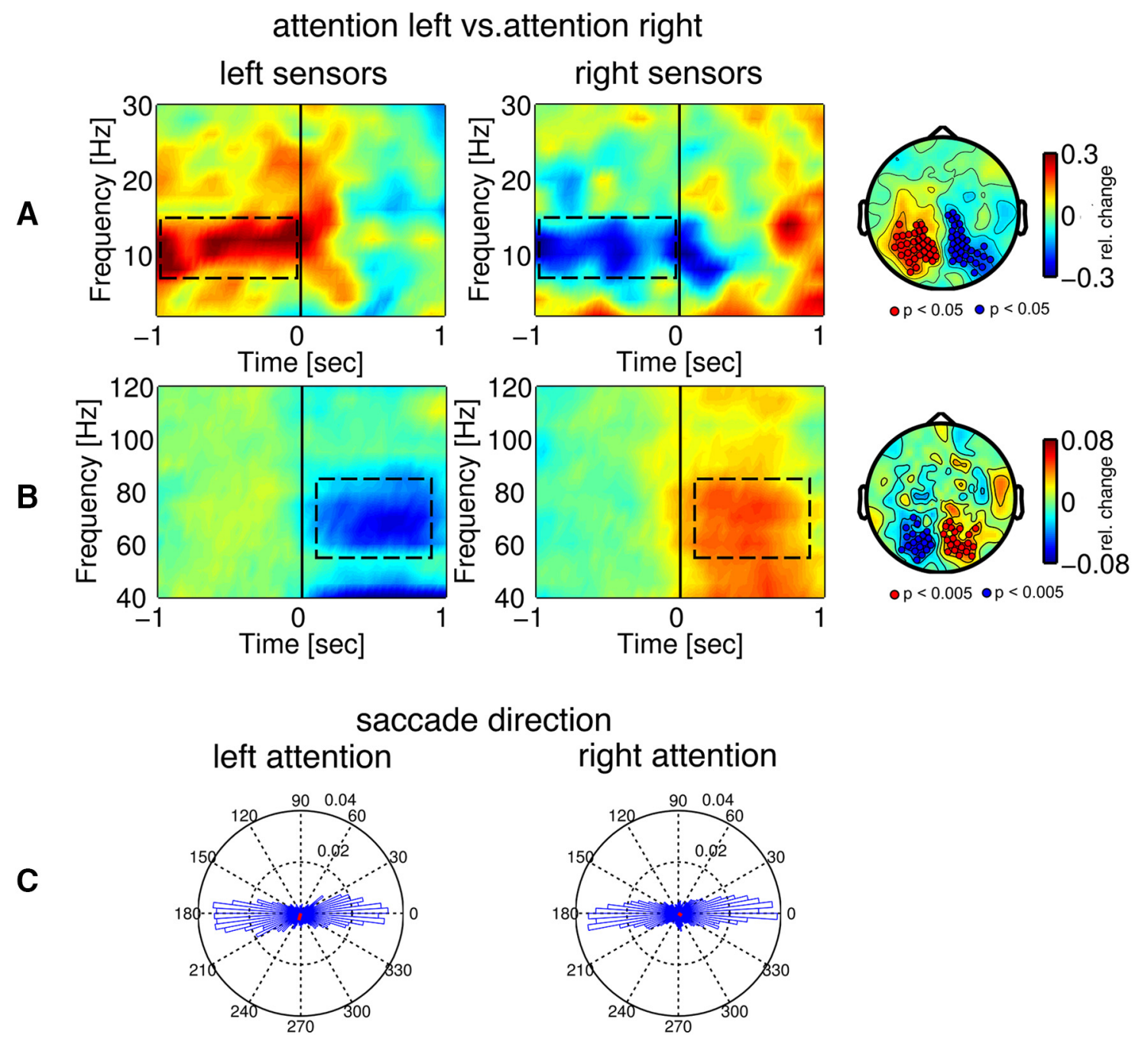

Figure 2. Modulations and interactions between low and high-frequency activity. Shown are TFRs of power aligned to target onset $(t=0 \mathrm{~s})$ for left versus right cued trials according to the following formula: relative power change $=\left(\right.$ power $_{\text {left attention }}-$ power $\left._{\text {right attention }}\right) /\left(\right.$ power $_{\text {left attention }}+$ power $\left._{\text {right attention }}\right)$. $A$, The alpha power increased in sensors ipsilateral to the cue direction, whereas it decreased in contralateral sensors. The topography (right) illustrates the $8-13 \mathrm{~Hz}$ alpha power modulation in the -1 to 0 s interval (dashed box). The marked sensors indicate significant clusters when controlling for multiple comparisons (left vs right attention). $\boldsymbol{B}$, As in $\boldsymbol{A}$, but for high-frequency modulation. The topography (right) illustrates the $60-80 \mathrm{~Hz}$ gamma power modulation in the 0.1- $0.9 \mathrm{~s}$ interval (dashed rectangles) after array onset. The gamma power decreased relatively in sensors ipsilateral to the cue direction, whereas it increased in contralateral sensors. C, Directional distribution of all saccades $\left(N_{\text {total left }}=17790 ; N_{\text {total }}\right.$ - right $\left.=15832\right)$ during the delay interval before target onset at $t=0$ s show a horizontal preference without significant differences (Kolmogorov-Smirnov test, $p>0.2$ ) between the distribution corresponding to the left and right attention conditions.

and Methods) was computed per trial and direction of attention by considering the anticipatory alpha-band activity ( -1 to $0 \mathrm{~s})$ in ipsilateral and contralateral sensors (marked in Fig. 2). The data were split into trials with high and low alpha lateralization indices. The left side of Figure $4 C$ illustrates that, independently of the direction of attention, trials can be split in two pools of high and low LI. Whereas the alpha LI per definition is different from zero, it is also the case for gamma for the same pools of trials. It is the deviation from zero for both alpha and gamma that is emphasized in Figure 4C. Importantly, trials dominated by strong alpha hemispheric lateralization during the delay period were characterized by strong gamma-band hemispheric lateralization upon stimulus presentation (Fig. $4 C$, right bars; left attention: $t_{(29)}=$ $-2.08, p<0.05$; right attention: $t_{(29)}=-2.8, p<0.01$ ). As an example, consider the trials in an individual in whom attention was cued to the left hemifield. In anticipation of a target in the cued left hemifield, alpha decreased in right sensors, whereas it increased relatively in left sensors. Upon target onset, gamma power increased in right sensors compared with the left sensors.
The degree of alpha lateralization was predictive of the gammaband lateralization across trials.

The degree of alpha lateralization in individual participants was also predictive of gamma lateralization; that is, it correlated negatively across participants (Fig. $4 D$; Spearman's rank-order correlation; $\rho=-0.52, p<0.01$ ). In summary, stronger modulation of alpha (delay interval) and gamma (stimulus processing) activity was related to behavioral outcome. Alpha power modulations during the delay interval were predictive for subsequent gamma power in both between and within participants.

\section{Right frontal eye field (rFEF) exerts top-down control over primary visual areas}

Given that alpha-band activity in early visual areas (Fig. 5A) is strongly modulated by the anticipatory allocation of attention toward the cued location, it appears to be under top-down control. To identify the regions driving the alpha-band activity, we applied a GC analysis. The time-domain signal was projected to "source space" using a beamformer technique. Signals from V1 


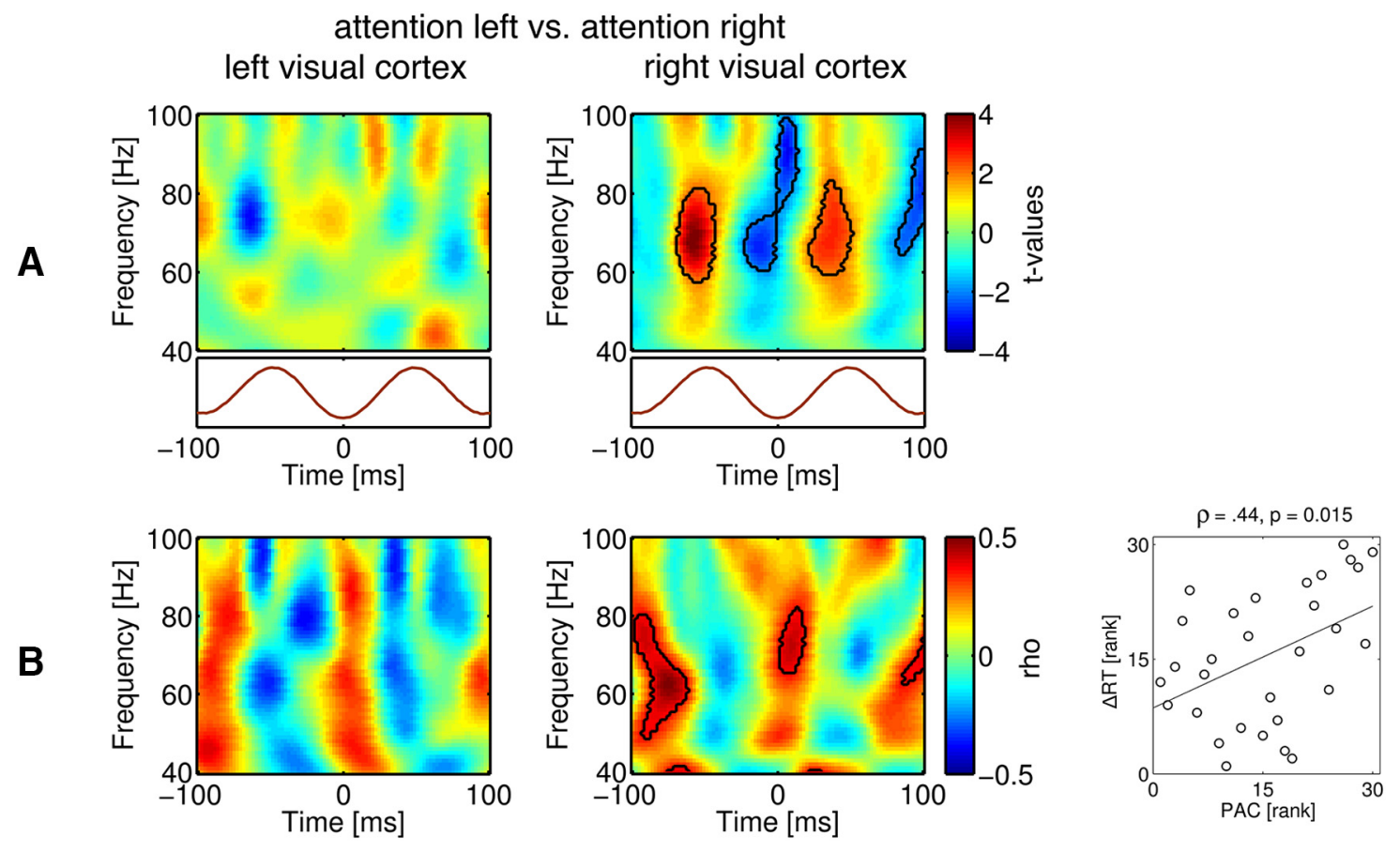

Figure 3. PAC as function of attention direction. $\boldsymbol{A}$, TFR of power phase aligned to the trough of the alpha activity during the delay interval. Traces at the bottom illustrate two cycles of the corresponding phase of the modulating rhythm. The color code indicates condition differences in coupling ( $t$ values; left vs right attention). Outlines highlight TFR clusters according to significance (cluster-based permutation test, $p<0.05$ ). Alpha to gamma cross-frequency coupling was more pronounced in sensors contralateral to the cued location. $\boldsymbol{B}$, Relationship between PAC and change in behavioral performance. Outlines highlight TFR clusters demonstrating a significant relationship. Scatterplot provides an additional view of the observed effect in which each dot represents a single subject.

were used as the reference signal on the basis of the peaks of reconstructed alpha power fluctuations (Fig. 5A). The directionality of the information flow was evaluated on the basis of a nonparametric pairwise GC analysis (Wen et al., 2013). The GC influence was computed for all locations to V1 (feedback) and V1 to all locations (feedforward). The GC influence was computed for left and right visual seeds and combined across participants. The difference between feedforward and feedback GC in the 8-13 $\mathrm{Hz}$ alpha band yielded a metric of the directionality. The strongest influence was found from $\mathrm{rFEF}$ (Fig. $5 B$, MR images) to bilateral V1 (Fig. 5A, MR images). This effect was dominant in the alpha band, as confirmed by the GC spectra (Fig. 5B; $p<0.05$ cluster-based permutation test). It is important to note that a clear $\sim 10 \mathrm{~Hz}$ peak was also evident in both FEFs when considering power alone; however, the power was not modulated by attention. Furthermore, the GC influence was independent of the direction of attention and seed regions (left and right V1), yielding similar parametric maps in all four conditions (left seed-left attention, left seed-right attention, right seed-left attention, and right seed-right attention). No GC effects in the gamma-band range were found. In summary, the Granger causality analysis suggests that one source of top-down control for the modulation of alpha-band activity observed in visual areas is the FEF.

\section{Discussion}

In the present study, subjects performed a version of the EriksenFlanker task while brain activity was acquired using MEG. Consistent with previous literature (Foxe et al., 1998; Sauseng et al., 2005; Kelly et al., 2006; Thut et al., 2006; Händel et al., 2011; Bauer et al., 2014; Lozano-Soldevilla et al., 2014; Marshall et al., 2015b), the allocation of spatial attention was associated with hemisphere-specific modulations of alpha activity. Upon target presentation, a complementary pattern of gamma activity was observed (Bauer et al., 2014; Lozano-Soldevilla et al., 2014; Marshall et al., 2015b). Stronger modulation of alpha and gamma activity was associated with enhanced ability to detect targets among flanking distracters. Importantly, decreases of anticipatory alpha activity across trials were predictive of stimulusinduced gamma-band activity. A spectral GC analysis, computed for the entire frequency spectrum including low- and highfrequency activity, showed that the visual alpha activity was under top-down control of the FEF.

\section{Alpha oscillatory activity precedes stimulus-induced gamma activity}

When operating in rich, natural environments, the convergence in the visual hierarchy introduces an information-processing problem. It has been hypothesized that attention mechanisms serve to prioritize and gate information selectively. Recent evidence from monkey electrophysiology makes a strong case that neuronal synchronization in the gamma band reflects the feedforward flow of information, whereas feedback control is exerted through synchronization of activity in the lower alpha and beta $(8-20 \mathrm{~Hz})$ frequency ranges (Saalmann et al., 2012; van Kerkoerle et al., 2014; Bastos et al., 2015). Previous reports have demonstrated that internal "cognitive" states such as anticipatory allocation of attention is associated with modulations of neuronal activity in sensory areas (Foxe et al., 1998; Kastner et al., 1999; Worden et al., 2000; Kelly et al., 2006; van Ede et al., 2010; Händel et al., 2011; van Ede et al., 2014). However, the neuronal mechanisms of this feedback are still under debate. The present results are consistent with the notion that parieto-occipital alpha oscillations are modulated by internal (top-down) mechanisms reflecting the anticipation of upcoming stimuli. Conversely, power increases in the gamma band are stimulus driven and amplified by the allocation of attention (Siegel et al., 2008; Koelewijn et al., 


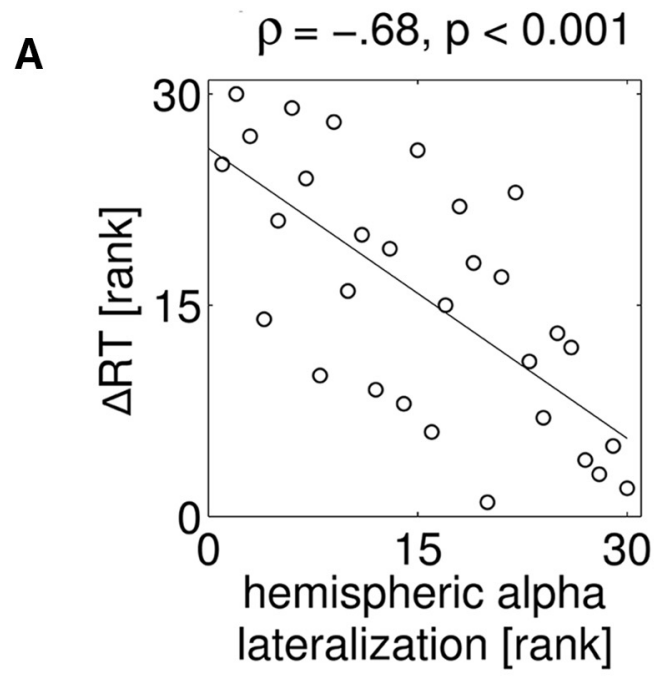

C
B

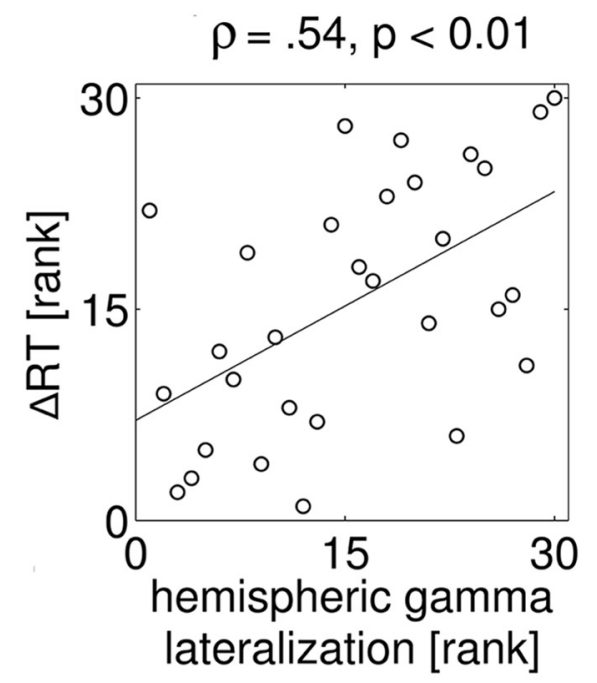

D
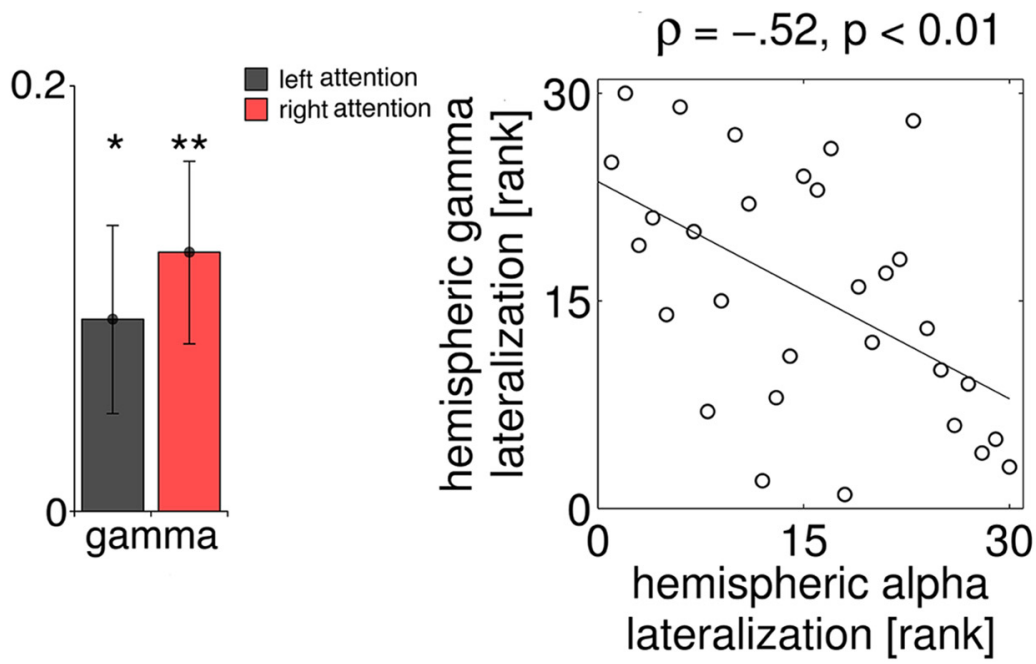

Figure 4. Relationship between anticipatory alpha power and stimulus-induced gamma power within and between participants. $\boldsymbol{A}$, Correlation between prestimulus hemisphere-specific alpha lateralization and the RT differences between IC and C trials. B, Correlation between gamma band hemisphere-specific lateralization and RT differences between IC and C trials. Participants who were less distracted by the IC flankers showed stronger alpha- and gamma-band power modulations. C, For each individual, the trials were divided in high and low alpha LI (LI_alpha) and subsequently averaged. Gray color indicates attention directed to the left hemifield and red colors attention directed to the right hemifield. For this trial division, the gamma LI (LI_gamma) was computed. The right bar graphs illustrate the difference (high - low), confirming that trials dominated by high prestimulus alpha lateralization were characterized by high stimulus-induced gamma lateralization. ${ }^{*} p<0.05 ;{ }^{* *} p<0.01$. D, Spearman's rank correlation coefficient analysis across subjects demonstrated a significant relationship between prestimulus alpha and stimulus-induced gamma lateralization.

2013; Lozano-Soldevilla et al., 2014). These effects were observed in similar clusters of sensors over parieto-occipital sites. Our findings demonstrate that the magnitude of prestimulus alpha lateralization is predictive of the magnitude of stimulus-induced gamma lateralization; that is, decreases in local alpha power are associated with and predictive of increases in local gamma power. Therefore, top-down control results in modulation of alpha activity that appears to exert gain control of stimulus-induced gamma activity. Importantly, the modulations in alpha and gamma power were correlated with behavioral performance. Together, these results suggest that changes in neuronal synchronization control, no just the routing of information during spatial allocation of attention, but also the degree to which individual subjects can use spatial attention successfully to filter unwanted information.
Even though the power of alpha oscillations was reduced during the allocation of attention, the oscillations were still functional because they were coupled to gamma activity, modulating its power in a phasic manner (Fig. 3). Concerns have been raised on measures of PAC because the metrics applied are sensitive to nonsinusoidal wave shapes (Aru et al., 2015). However, we find the PAC to increase when attention is on and alpha power is low. This reduces concerns that the PAC that we report is explained by spurious effects (Jensen et al., 2016). Furthermore, in the context of the present experimental design, it is not possible to address how the prestimulus alpha phase modulates poststimulus gamma because the stimulus-evoked response would be confounded by power in the gamma band. Our results corroborate findings from nonhuman primates showing alpha-gamma cross-frequency coupling in the pulvinar during the delay period of the same 
A
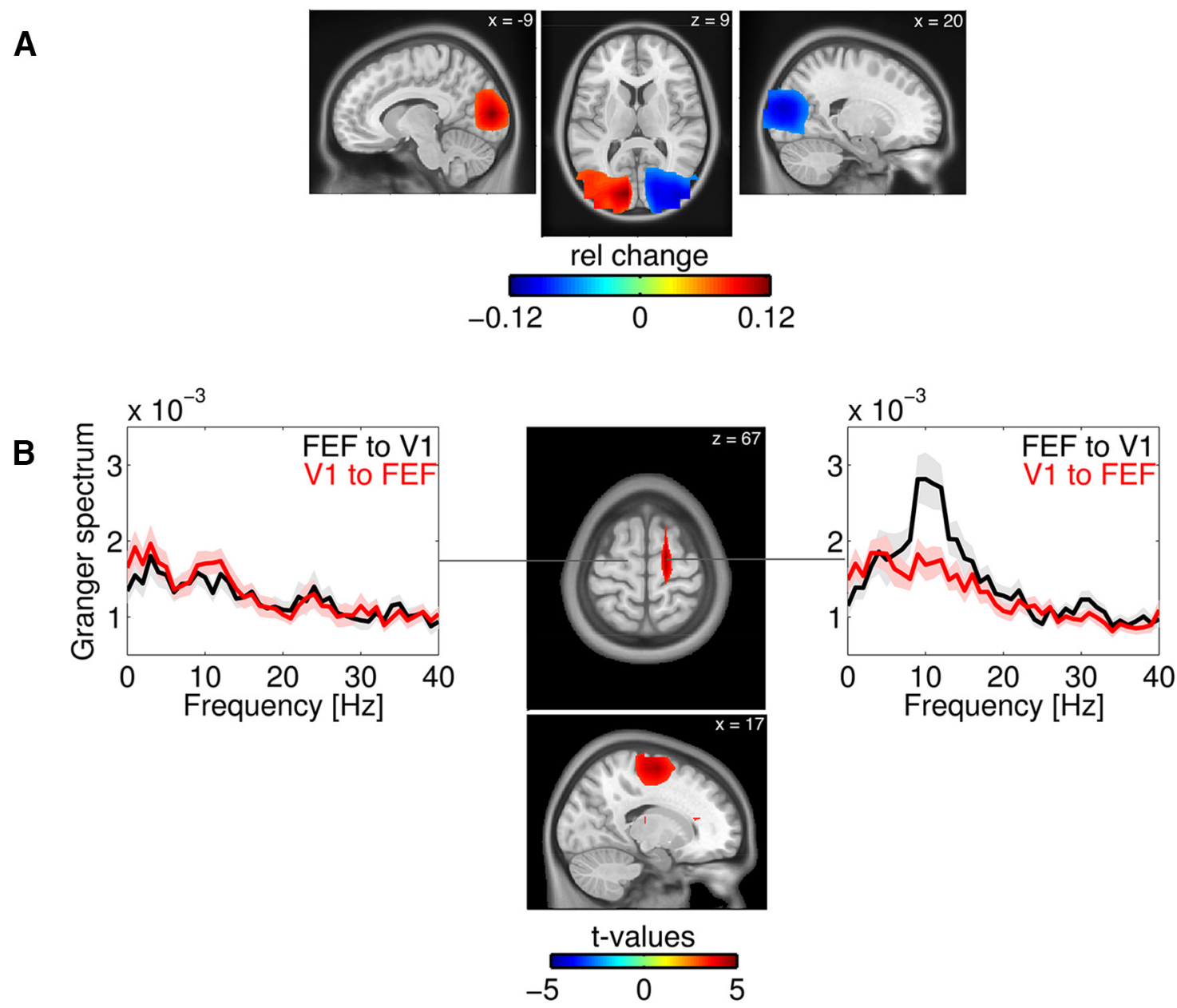

Figure 5. Early visual areas are under top-down control by FEF as reflected by modulations of alpha-band activity. $A$, Sources of the alpha power modulation $(t=-1$ to $0 \mathrm{~s} ; f=8-13 \mathrm{~Hz})$ with respect to left versus right cues were found in $\mathrm{V} 1$ (thresholded at $p<0.05$ after permutation approach). Signals from these locations were used as seeds in the $\mathrm{GC}$ analysis. $\boldsymbol{B}, \mathrm{GC}$ spectra (left and right plots) separately for the top-down (black traces) and bottom-up (red traces) control. GC spectra were calculated from the left and rFEF, respectively. Whole-brain analysis contrasting "All to V1" vs "V1 to All" confirmed a significant top-down drive of V1 by the rFEF. $p<0.05$, cluster-based random permutation procedure.

Flanker task (Saalmann et al., 2012). It has been proposed that decreases in amplitude might enlarge the duty cycle of the alpha rhythm, which allows for longer bursts of gamma activity (Jensen et al., 2014). Therefore, upon cue presentation, the locationspecific widening of the duty cycle will allow for stronger gammaband activity. Therefore, visual processing may be enhanced, which may result in an increased ability to process visual targets in the presence of distracters.

\section{Alpha activity in early sensory areas is under top-down control by a phasic drive from the FEF}

Previous brain-imaging studies in humans and electrophysiology studies in monkeys have demonstrated that early sensory areas can be influenced by frontoparietal areas during spatial attention (Kastner et al., 1999; Capotosto et al., 2009; Marshall et al., 2015a; Marshall et al., 2015b). In particular, it has been shown that the strength of the anatomical connections between frontal and posterior areas predicts modulation of alpha and gamma activity in sensory areas in individual subjects (Marshall et al., 2015a). Moreover, it has been shown that interfering with neural activity in FEF by means of repetitive transcranial magnetic stimulation (TMS) leads to a disruption of anticipatory visual alpha activity (Capotosto et al., 2009; Marshall et al., 2015b). On the basis of whole-brain analysis, our results suggest that the FEF exerts a phasic modulation of the visual alpha activity. It has been reported that FEF has direct projections to visual cortical areas V1 and V4 (Barone et al., 2000; Markov and Kennedy, 2013). This finding extends the monkey physiology (van Kerkoerle et al., 2014; Bastos et al., 2015) and recent observations in humans (Michalareas et al., 2016; Wang et al., 2016) in demonstrating a possible mechanism by which specifically the FEF may exert topdown control; that is, by modulating low-frequency oscillations in sensory areas. In our study, only the rFEF appeared to exert a top-down influence on visual areas, which may be indicative of a suggested right hemispheric FEF dominance of spatial attention control (Grosbras and Paus, 2003; Silvanto et al., 2006; Capotosto et al., 2009; Duecker et al., 2013; Cazzoli et al., 2015; Chanes et al., 2015; Marshall et al., 2015b; Wang et al., 2016). A right hemispheric dominance of attention function is well known from the clinical literature on visuospatial hemi-neglect (Mesulam, 1999; Heilman et al., 2000) and stronger right hemispheric activation is often observed in human fMRI studies of attention (Szczepanski et al., 2010). In particular, when spatial attention is reoriented, right brain structures around the temporoparietal junction are engaged (Corbetta et al., 2008). Such biases in lateralization could partly explain the present findings demonstrating that PAC and GC are more strongly modulated by attention in the right than left hemisphere. However, such a conclusion should be made 
with caution. First, a model of right hemispheric FEF dominance in spatial attention control would imply that the rFEF contains a map of the entire visual field. Functional brain-imaging studies, however, do not support such an assumption and show instead a topographic representation that is biased toward the contralateral hemifield (Kastner et al., 2007; Silver and Kastner, 2009; Szczepanski et al., 2010; Szczepanski and Kastner, 2013). Second, functional brain imaging and TMS studies have thus far supported a hemispheric competition model of spatial attention control in which frontoparietal cortex of each hemisphere controls the contralateral hemifield rather than a hemispheric dominance model (Neggers et al., 2007; Sauseng et al., 2011; Marshall et al., 2015b). Third, such conclusion would be primarily based on a negative finding in the left FEF, which might be explained by inadequate signal-to-noise ratio. Another important consideration is that we were only able to measure activity from cortical generators, so all of our assumptions regarding the feedforward and feedback information flow are limited to corticocortical interactions. As shown in recent monkey physiology studies (Saalmann et al., 2012; Zhou et al., 2016), it is possible that thalamic regions such as the pulvinar play an important role in coordinating the routing of information between neocortical networks.

In summary, the present study suggests two novel aspects of alpha oscillatory activity in organizing functional brain architecture: exerting control of upcoming gamma-band activity and enabling frontal feedback control. Therefore, feedforward and feedback mechanisms appear to rely on different frequency bands. This is consistent not only at the macroscopic level, but also when considering cortical laminae. Infragranular layers are reported as the primary generators of the alpha activity (Bollimunta et al., 2011; Buffalo et al., 2011; Maier et al., 2011; Dougherty et al., 2015), whereas superficial layers seem to be dominated by gamma activity (Maier et al., 2010; Buffalo et al., 2011). In addition, alpha activity in deep layers has been found to exert phasic modulation of the gamma activity in superficial layers (Spaak et al., 2012), as well as to regulate spiking activity evoked by visual stimuli (Dougherty et al., 2015). In future work, it will be of great relevance to unravel the cross-frequency interactions of alpha/beta- and gamma-band activity to determine by which neuronal mechanisms in relation to cortical layers these interactions mediate the integration of feedback and feedforward information. The functional specificity of the FEF-V1 connectivity and its interaction with other brain areas should also be addressed in future investigations. The FEFs are certainly not the only relevant structures of the frontoparietal network that are involved with the control of spatial attention. Subcortical structures such as the striatum or the pulvinar nucleus have been proposed to coordinate neocortical rhythmicity (Tort et al., 2008; Saalmann et al., 2012; Horschig et al., 2015; Zhou et al., 2016). Future work obtaining activity from an extensive cortical and subcortical network will further our understanding of the dynamic routing of information as governed by feedforward and feedback relationships.

\section{References}

Aru J, Aru J, Priesemann V, Wibral M, Lana L, Pipa G, Singer W, Vicente R (2015) Untangling cross-frequency coupling in neuroscience. Curr Opin Neurobiol 31:51-61. CrossRef Medline

Bahramisharif A, van Gerven M, Heskes T, Jensen O (2010) Covert attention allows for continuous control of brain-computer interfaces. Eur J Neurosci 31:1501-1508. CrossRef Medline

Barone P, Batardiere A, Knoblauch K, Kennedy H (2000) Laminar distribution of neurons in extrastriate areas projecting to visual areas V1 and V4 correlates with the hierarchical rank and indicates the operation of a distance rule. J Neurosci 20:3263-3281. Medline

Bastiaansen MC, Knösche TR (2000) Tangential derivative mapping of axial MEG applied to event-related desynchronization research. Clin Neurophysiol 111:1300-1305. CrossRef Medline

Bastos AM, Schoffelen JM (2015) A tutorial review of functional connectivity analysis methods and their interpretational pitfalls. Front Syst Neurosci 9:175. CrossRef Medline

Bastos AM, Vezoli J, Bosman CA, Schoffelen JM, Oostenveld R, Dowdall JR, De Weerd P, Kennedy H, Fries P (2015) Visual areas exert feedforward and feedback influences through distinct frequency channels. Neuron 85:390-401. CrossRef Medline

Bauer M, Stenner MP, Friston KJ, Dolan RJ (2014) Attentional modulation of alpha/beta and gamma oscillations reflect functionally distinct processes. J Neurosci 34:16117-16125. CrossRef Medline

Bollimunta A, Mo J, Schroeder CE, Ding M (2011) Neuronal mechanisms and attentional modulation of corticothalamic alpha oscillations. J Neurosci 31:4935-4943. CrossRef Medline

Buffalo EA, Fries P, Landman R, Buschman TJ, Desimone R (2011) Laminar differences in gamma and alpha coherence in the ventral stream. Proc Natl Acad Sci U S A 108:11262-11267. CrossRef Medline

Capotosto P, Babiloni C, Romani GL, Corbetta M (2009) Frontoparietal cortex controls spatial attention through modulation of anticipatory alpha rhythms. J Neurosci 29:5863-5872. CrossRef Medline

Cazzoli D, Jung S, Nyffeler T, Nef T, Wurtz P, Mosimann UP, Müri RM (2015) The role of the right frontal eye field in overt visual attention deployment as assessed by free visual exploration. Neuropsychologia 74: 37-41. CrossRef Medline

Chanes L, Quentin R, Vernet M, Valero-Cabré A (2015) Arrhythmic activity in the left frontal eye field facilitates conscious visual perception in humans. Cortex 71:240-247. CrossRef Medline

Corbetta M, Patel G, Shulman GL (2008) The reorienting system of the human brain: from environment to theory of mind. Neuron 58:306 -324. CrossRef Medline

Ding M, Chen Y, Bressler S (2006) Granger causality: basic theory and application to neuroscience. In: Handbook of time series analysis (Schelter B, Winterhalder M, Timmer J, eds), pp 451-474. Weinheim, Germany: Wiley-VCH.

Dougherty K, Cox MA, Ninomiya T, Leopold DA, Maier A (2017) Ongoing alpha activity in V1 regulates visually driven spiking responses. Cereb Cortex 27:1113-1124. CrossRef

Duecker F, Formisano E, Sack AT (2013) Hemispheric differences in the voluntary control of spatial attention: direct evidence for a righthemispheric dominance within frontal cortex. J Cogn Neurosci 25:13321342. CrossRef Medline

Engbert R, Kliegl R (2003) Microsaccades uncover the orientation of covert attention. Vision Res 43:1035-1045. CrossRef Medline

Eriksen BA, Eriksen CW (1974) Effects of noise letters upon the identification of a target letter in a nonsearch task. Percept Psychophysiol 16:143149. CrossRef

Eriksen CW (1995) The flankers task and response competition: a useful tool for investigating a variety of cognitive problems. Vis Cogn 2:101-118. CrossRef

Foxe JJ, Simpson GV, Ahlfors SP (1998) Parieto-occipital approximately 10 $\mathrm{Hz}$ activity reflects anticipatory state of visual attention mechanisms. Neuroreport 9:3929-3933. CrossRef Medline

Fries P, Reynolds JH, Rorie AE, Desimone R (2001) Modulation of oscillatory neuronal synchronization by selective visual attention. Science 291: 1560-1563. CrossRef Medline

Granger CWJ (1969) Investigating causal relations by econometric models and cross-spectral methods. Econometrica 37:424-438. CrossRef

Grosbras MH, Paus T (2003) Transcranial magnetic stimulation of the human frontal eye field facilitates visual awareness. Eur J Neurosci 18:31213126. CrossRef Medline

Gross J, Kujala J, Hamalainen M, Timmermann L, Schnitzler A, Salmelin R (2001) Dynamic imaging of coherent sources: Studying neural interactions in the human brain. Proc Natl Acad Sci U S A 98:694-699. CrossRef Medline

Hämäläinen M, Hari R, Ilmoniemi RJ, Knuutila J, Lounasmaa OV (1993) Magnetoencephalography-theory, instrumentation, and applications to noninvasive studies of the working human brain. Rev Mod Phys 65:413497. CrossRef 
Händel BF, Haarmeier T, Jensen O (2011) Alpha oscillations correlate with the successful inhibition of unattended stimuli. J Cogn Neurosci 23: 2494-2502. CrossRef Medline

Heilman KM, Valenstein E, Watson RT (2000) Neglect and related disorders. Semin Neurol 20:463-470. CrossRef Medline

Horschig JM, Smolders R, Bonnefond M, Schoffelen JM, van den Munckhof P, Schuurman PR, Cools R, Denys D, Jensen O (2015) Directed communication between nucleus accumbens and neocortex in humans is differentially supported by synchronization in the theta and alpha band. PLoS One 10:e0138685. CrossRef Medline

Jensen O, Gips B, Bergmann TO, Bonnefond M (2014) Temporal coding organized by coupled alpha and gamma oscillations prioritize visual processing. Trends Neurosci 37:357-369. CrossRef Medline

Jensen O, Spaak E, Park H (2016) Discriminating valid from spurious indices of phase-amplitude coupling. eNeuro 3: pii: ENEURO.0334-16.2016. CrossRef Medline

Jung TP, Makeig S, McKeown MJ, Bell AJ, Lee TW, Sejnowski TJ (2001) Imaging brain dynamics using independent component analysis. Proc IEEE Inst Electr Electron Eng 89:1107-1122. CrossRef Medline

Kastner S, Pinsk MA, De Weerd P, Desimone R, Ungerleider LG (1999) Increased activity in human visual cortex during directed attention in the absence of visual stimulation. Neuron 22:751-761. CrossRef Medline

Kastner S, DeSimone K, Konen CS, Szczepanski SM, Weiner KS, Schneider KA (2007) Topographic maps in human frontal cortex revealed in memory-guided saccade and spatial working-memory tasks. J Neurophysiol 97:3494-3507. CrossRef Medline

Kelly SP, Lalor EC, Reilly RB, Foxe JJ (2006) Increases in alpha oscillatory power reflect an active retinotopic mechanism for distracter suppression during sustained visuospatial attention. J Neurophysiol 95:3844-3851. CrossRef Medline

Koelewijn L, Rich AN, Muthukumaraswamy SD, Singh KD (2013) Spatial attention increases high-frequency gamma synchronisation in human medial visual cortex. Neuroimage 79:295-303. CrossRef Medline

Liu Y, Bengson J, Huang H, Mangun GR, Ding M (2016) Top-down modulation of neural activity in anticipatory visual attention: control mechanisms revealed by simultaneous EEG-fMRI. Cereb Cortex 26:517-529. CrossRef Medline

Lozano-Soldevilla D, ter Huurne N, Cools R, Jensen O (2014) GABAergic modulation of visual gamma and alpha oscillations and its consequences for working memory performance. Curr Biol 24:2878-2887. CrossRef Medline

Maier A, Adams GK, Aura C, Leopold DA (2010) Distinct superficial and deep laminar domains of activity in the visual cortex during rest and stimulation. Front Syst Neurosci 4: pii: 31. CrossRef Medline

Maier A, Aura CJ, Leopold DA (2011) Infragranular sources of sustained local field potential responses in macaque primary visual cortex. J Neurosci 31:1971-1980. CrossRef Medline

Maris E, Oostenveld R (2007) Nonparametric statistical testing of EEG- and MEG-data. J Neurosci Methods 164:177-190. CrossRef Medline

Markov NT, Kennedy H (2013) The importance of being hierarchical. Curr Opin Neurobiol 23:187-194. CrossRef Medline

Marshall TR, Bergmann TO, Jensen O (2015a) Frontoparietal structural connectivity mediates the top-down control of neuronal synchronization associated with selective attention. PLoS Biol 13:e1002272. CrossRef Medline

Marshall TR, O'Shea J, Jensen O, Bergmann TO (2015b) Frontal eye fields control attentional modulation of alpha and gamma oscillations in contralateral occipitoparietal cortex. J Neurosci 35:1638-1647. CrossRef Medline

Martin A, Wang L, Saalmann Y, Shestyuk A, Jeong SK, Crone N, Parvizi J, Knight R, Kastner S (2015) Modulation of intracranial field potential responses in the human large-scale attention network during a spatial attention task. J Vis 15:1055. CrossRef

Mesulam MM (1999) Spatial attention and neglect: parietal, frontal and cingulate contributions to the mental representation and attentional targeting of salient extrapersonal events. Philos Trans R Soc Lond B Biol Sci 354:1325-1346. CrossRef Medline

Michalareas G, Vezoli J, van Pelt S, Schoffelen JM, Kennedy H, Fries P (2016) Alpha-beta and gamma rhythms subserve feedback and feedforward influences among human visual cortical areas. Neuron 89:384-397. CrossRef Medline

Mitra PP, Pesaran B (1999) Analysis of dynamic brain imaging data. Biophys J 76:691-708. CrossRef Medline

Müller MM, Gruber T, Keil A (2000) Modulation of induced gamma-band activity in the human EEG by attention and visual information processing. Int J Psychophysiol 38:283-299. CrossRef Medline

Neggers SF, Huijbers W, Vrijlandt CM, Vlaskamp BN, Schutter DJ, Kenemans JL (2007) TMS pulses on the frontal eye fields break coupling between visuospatial attention and eye movements. J Neurophysiol 98: 2765-2778. CrossRef Medline

Nolte G (2003) The magnetic lead field theorem in the quasi-static approximation and its use for magnetoencephalography forward calculation in realistic volume conductors. Phys Med Biol 48:3637-3652. CrossRef Medline

Oostenveld R, Fries P, Maris E, Schoffelen JM (2011) FieldTrip: Open source software for advanced analysis of MEG, EEG, and invasive electrophysiological data. Comput Intell Neurosci 2011:156869. CrossRef Medline

Palva S, Palva JM (2007) New vistas for alpha-frequency band oscillations. Trends Neurosci 30:150-158. CrossRef Medline

Palva S, Palva JM (2011) Functional roles of alpha-band phase synchronization in local and large-scale cortical networks. Front Psychol 2:204. CrossRef Medline

Pfurtscheller G (1977) Graphical display and statistical evaluation of eventrelated desynchronization (ERD). Electroencephalogr Clin Neurophysiol 43:757-760. CrossRef Medline

Pfurtscheller G, Aranibar A (1979) Evaluation of event-related desynchronization (ERD) preceding and following voluntary self-paced movement. Electroencephalogr Clin Neurophysiol 46:138-146. CrossRef Medline

Rihs TA, Michel CM, Thut G (2007) Mechanisms of selective inhibition in visual spatial attention are indexed by alpha-band EEG synchronization. Eur J Neurosci 25:603-610. CrossRef Medline

Saalmann YB, Pinsk MA, Wang L, Li X, Kastner S (2012) The pulvinar regulates information transmission between cortical areas based on attention demands. Science 337:753-756. CrossRef Medline

Sauseng P, Klimesch W, Stadler W, Schabus M, Doppelmayr M, Hanslmayr S, Gruber WR, Birbaumer N (2005) A shift of visual spatial attention is selectively associated with human EEG alpha activity. Eur J Neurosci 22:2917-2926. CrossRef Medline

Sauseng P, Feldheim JF, Freunberger R, Hummel FC (2011) Right prefrontal TMS disrupts interregional anticipatory EEG alpha activity during shifting of visuospatial attention. Front Psychol 2:241. CrossRef Medline

Siegel M, Donner TH, Oostenveld R, Fries P, Engel AK (2008) Neuronal synchronization along the dorsal visual pathway reflects the focus of spatial attention. Neuron 60:709-719. CrossRef Medline

Silvanto J, Lavie N, Walsh V (2006) Stimulation of the human frontal eye fields modulates sensitivity of extrastriate visual cortex. J Neurophysiol 96:941-945. CrossRef Medline

Silver MA, Kastner S (2009) Topographic maps in human frontal and parietal cortex. Trends Cogn Sci 13:488-495. CrossRef Medline

Spaak E, Bonnefond M, Maier A, Leopold DA, Jensen O (2012) Layerspecific entrainment of gamma-band neural activity by the alpha rhythm in monkey visual cortex. Curr Biol 22:2313-2318. CrossRef Medline

Spaak E, de Lange FP, Jensen O (2014) Local entrainment of alpha oscillations by visual stimuli causes cyclic modulation of perception. J Neurosci 34:3536-3544. CrossRef Medline

Szczepanski SM, Kastner S (2013) Shifting attentional priorities: control of spatial attention through hemispheric competition. J Neurosci 33:54115421. CrossRef Medline

Szczepanski SM, Konen CS, Kastner S (2010) Mechanisms of spatial attention control in frontal and parietal cortex. J Neurosci 30:148-160. CrossRef Medline

Tallon-Baudry C, Bertrand O (1999) Oscillatory gamma activity in humans and its role in object representation. Trends Cogn Sci 3:151-162. CrossRef Medline

Thut G, Nietzel A, Brandt SA, Pascual-Leone A (2006) Alpha-band electroencephalographic activity over occipital cortex indexes visuospatial attention bias and predicts visual target detection. J Neurosci 26:9494-9502. CrossRef Medline

Tiesinga PH, Fellous JM, Salinas E, Jose JV, Sejnowski TJ (2004) Synchronization as a mechanism for attentional gain modulation. Neurocomputing 58-60:641-646.

Tort AB, Kramer MA, Thorn C, Gibson DJ, Kubota Y, Graybiel AM, Kopell NJ (2008) Dynamic cross-frequency couplings of local field potential oscillations in rat striatum and hippocampus during performance of a T-maze task. Proc Natl Acad Sci U S A 105:20517-20522. CrossRef Medline

van Ede F, Jensen O, Maris E (2010) Tactile expectation modulates pre- 
stimulus beta-band oscillations in human sensorimotor cortex. Neuroimage 51:867-876. CrossRef Medline

van Ede F, Szebényi S, Maris E (2014) Attentional modulations of somatosensory alpha, beta and gamma oscillations dissociate between anticipation and stimulus processing. Neuroimage 97:134-141. CrossRef Medline

van Kerkoerle T, Self MW, Dagnino B, Gariel-Mathis MA, Poort J, van der Togt C, Roelfsema PR (2014) Alpha and gamma oscillations characterize feedback and feedforward processing in monkey visual cortex. Proc Natl Acad Sci U S A 111:14332-14341. CrossRef Medline

Van Veen BD, van Drongelen W, Yuchtman M, Suzuki A (1997) Localization of brain electrical activity via linearly constrained minimum variance spatial filtering. IEEE Trans Biomed Eng 44:867-880. CrossRef Medline Wang C, Rajagovindan R, Han SM, Ding M (2016) Top-down control of visual alpha oscillations: sources of control signals and their mechanisms of action. Front Hum Neurosci 10:15. CrossRef Medline

Watrous AJ, Deuker L, Fell J, Axmacher N (2015) Phase-amplitude coupling supports phase coding in human ECoG. eLife 4.

Wen X, Rangarajan G, Ding M (2013) Multivariate Granger causality: an estimation framework based on factorization of the spectral density matrix. Philos Trans A Math Phys Eng Sci 371:20110610. CrossRef Medline

Worden MS, Foxe JJ, Wang N, Simpson GV (2000) Anticipatory biasing of visuospatial attention indexed by retinotopically specific alpha-band electroencephalography increases over occipital cortex. J Neurosci 20:RC63. Medline

Zhou H, Schafer RJ, Desimone R (2016) Pulvinar-cortex interactions in vision and attention. Neuron 89:209-220. CrossRef Medline 